\title{
Combined MMSE-PIC in Coded OFDM-CDMA Systems
}

\author{
Volker Kühn \\ Universität Bremen (Germany) \\ Department of Communications Engineering \\ Kufsteiner Strasse NW 1, D-28359 Bremen
}

\begin{abstract}
In this paper the performance of linear multi-user detection (MUD) and nonlinear parallel interference cancellation (PIC) is analyzed for a quasi synchronous OFDM-CDMA uplink transmission. Specifically, we investigate two different approaches concerning the combination of linear MUD techniques such as the decorrelator and the MMSE approach with nonlinear parallel interference cancellation (PIC). It is pointed out that OFDM-CDMA systems offer great advantages over single carrier systems due to flat fading conditions on each subcarrier leading to much lower implementation costs of MUD techniques.

Assuming perfectly known channel impulse responses for each user and a rough synchronization it turns out that the application of the MMSE-MUD filter leads to an insufficient equalization of the channel. Therefore, we propose an approach where the PIC loop works directly on the channel output and the MMSE filter is only active in the initial iteration loop. With this approach, the combination of linear MMSE-MUD and PIC shows excellent performance even for very high system loads.
\end{abstract}

\section{INTRODUCTION}

Code Division Multiple Access (CDMA) has been chosen in various modern communication systems [1], [2], [3] as multiple access technique. In this paper, the uplink of a multicarrier CDMA (MC-CDMA) system [4], [5] is considered using OFDM (Orthogonal Frequency Division Multiplex) to combat the frequency selectivity of the mobile radio channel. Therefore, each subcarrier is affected by flat fading and a one tap equalizer suffices for eliminating channel distortion.

In contrast to a synchronous downlink transmission where orthogonal spreading sequences suppress multi-user interference (MUI) efficiently, this orthogonality would be destroyed in an asynchronous uplink transmission. Therefore, pseudonoise (PN) sequences are used and multi-user interference is the limiting factor concerning system capacity.

In order to achieve high spectral efficiencies, the interference has to be attacked by multi-user detection (MUD) techniques. In the last years, plenty of work has been spent on multi-user detection [6], [7], [8], [9], [10], [11]. Capacity bounds have been analytically derived for different MUD techniques indicating the maximum system load that should be reachable in theory [8], [12], [13]. Furthermore, a lot of simulations have been carried out for single carrier systems operating in frequency non-selective and even frequency selective environments. In the latter case, MUD algorithms incorporate channel equalizer [14] resulting in high computational costs.

The aim of this paper is to analyze the performance of linear and nonlinear MUD exploiting the characteristics of coded OFDM-CDMA systems. One specific characteristic of OFDM-CDMA is the one-tap-equalization due to flat fading on each subcarrier. This enables the application of MUD algo- rithms developed for frequency non-selective channels saving valuable implementation costs when compared to frequency selective fading and single carrier systems. Specifically, the combination of linear MUD and nonlinear parallel interference cancellation is investigated.

The paper is structured as follows: Section 2 describes the OFDM-CDMA system with FEC coding and single-user detection (SUD). Next, section 3 presents the considered MUD techniques, their application in an OFDM-CDMA environment and discusses the obtained simulation results. Finally, section 4 gives some conclusions.

\section{SYSTEM DESCRIPTION}

Fig. 1 depicts the structure of the considered OFDM-CDMA transmitter. The information bits $d^{(j)}(k)$ of duration $T_{d}$ for each user $1 \leq j \leq J$ are encoded by a conventional convolutional code of rate $R_{c}=1 / n$. After encoding, the resulting vector $\mathbf{b}^{(j)}(k)$ is spread by repeating each coded bit $b_{i}^{(j)}(k)$, $1 \leq i \leq n, N_{p}$ times and successive multiplication with a user-specific code $\mathbf{c}^{(j)}$. Due to an asynchronous transmission in the uplink, we use simple pseudo-noise (PN) sequences for spreading. Throughout the paper, the duration of a chip $c_{\mu}^{(j)}$ equals $T_{c}=T_{d} / G_{p}$ where $G_{p}=N_{p} / R_{c}=64$ is the entire processing gain.

Next, the OFDM transmitter transforms the obtained vector $\tilde{\mathbf{b}}^{(j)}$ into the time domain. In this work, the number of carriers $N_{c}$ equals exactly the processing gain, i.e. one information bit $d^{(j)}(k)$ is mapped exactly onto one OFDM symbol. After frequency-domain interleaving $\left(\Pi_{f}\right)$ over $N_{c}$ chip and inverse Fourier transformation, a cyclic prefix of duration $T_{g}$ called guard interval is inserted in front of each OFDM symbol. The resulting signals $\mathbf{s}^{(j)}(k)$ of different users are

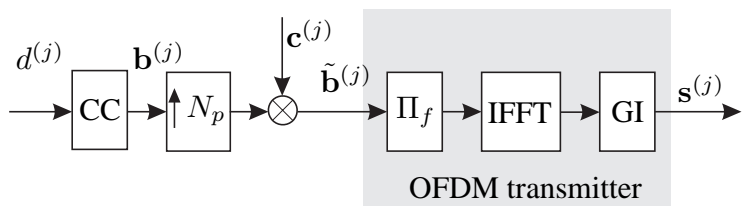

Fig. 1. Typical structure of an OFDM-CDMA transmitter

now transmitted over $J$ individual $L$-path mobile radio channels. Real and imaginary parts of the corresponding channel coefficients $h_{l}^{(j)}(k), 0 \leq l<L$, are gaussian distributed and statistically independent. Although each user is assigned to an individual channel, the number of transmission paths $L$ is as- 
sumed to be the same for all users. The corresponding transfer function is defined by

$$
H_{\mu}^{(j)}(k)=\sum_{l=0}^{L-1} h_{l}^{(j)}(k) \cdot e^{-j 2 \pi \mu l / L} .
$$

At the OFDM receiver, the cyclic prefix is removed first (Fig. 2). A guard time $T_{g}$ larger than the delay-spread $\Delta \tau$ of the channel results in a cyclic convolution of channel impulse response and transmitted signal. This enables us to efficiently transform the received signal back into the frequency domain by the fast Fourier transform (FFT). Assuming rough synchronization, i.e. the maximum delay between different users is limited to $T_{g}-\Delta \tau$, one FFT window suffices for transforming all user signals back into the frequency domain. The cyclic convolution in time domain corresponds to a scalar multiplication of $H_{\mu}^{(j)}(k)$ with the spread signal $\tilde{b}_{\mu}^{(j)}(k)$ in the frequency domain. Hence, this leads to an equivalent channel model where each chip is only affected by flat fading.

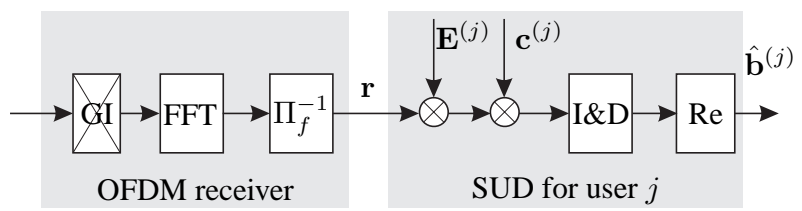

Fig. 2. Single-user receiver for OFDM-CDMA

The received vector $\mathbf{r}(k)$ at the output of the OFDM receiver at time instance $k$ consists of $N_{c}$ chips and has the form

$$
\mathbf{r}(k)=\mathbf{A}(k) \mathbf{b}(k)+\mathbf{n}(k)
$$

where

$$
\mathbf{b}(k)=\left(\mathbf{b}^{(1)}(k)^{T} \mathbf{b}^{(2)}(k)^{T} \ldots \mathbf{b}^{(J)}(k)^{T}\right)^{T}
$$

contains the convolutionally encoded bits $b_{i}^{(j)}(k)$ of all users and $\mathbf{n}(k)$ determines the background noise. The system matrix $\mathbf{A}(k)=\left(\mathbf{A}^{(1)}(k) \cdots \mathbf{A}^{(J)}(k)\right)$ comprises $J$ user specific matrices

$$
\mathbf{A}^{(j)}(k)=\left(\begin{array}{lll}
\mathbf{a}_{1}^{(j)}(k) & & \\
& \ddots & \\
& & \mathbf{a}_{n}^{(j)}(k)
\end{array}\right)
$$

where the column vectors have the form

$$
\mathbf{a}_{i}^{(j)}(k)=\left(a_{i, 0}^{(j)}(k) \cdots a_{i, N_{p}-1}^{(j)}(k)\right)^{T} .
$$

Its elements

$$
a_{i, \nu}^{(j)}(k)=c_{(i-1) N_{p}+\nu}^{(j)} H_{(i-1) N_{p}+\nu}^{(j)}(k), \quad 0 \leq \nu<N_{p}
$$

are element-wise products of the signature sequences $\mathbf{c}^{(j)}$ and the channel transfer function $\mathbf{H}^{(j)}(k)$. The influence of the interleaver $\Pi_{f}$ on the indices is neglected. The special form of $\mathbf{A}$ is caused by the specific mapping of the coded bits onto the OFDM symbols. Due to the fact that generally $n$ coded bits $b_{i}^{(j)}(k)$ are mapped onto one OFDM symbol, $\mathbf{A}$ is composed by $n J$ column vectors $\mathbf{a}_{i}^{(j)}(k)$. The rough synchronization mentioned before ensures that vectors with different indices $i$ do not mutually interfere. Therefore, $\mathbf{A}$ can be split up into $n$ different sub-matrices saving computational costs when calculating its pseudo-inverse for linear MUD.

The optimal single-user detection (SUD) employs a matched filter that maximizes the signal-to-noise ratio at its output. Presupposing perfectly known channel impulse responses, the equalizer $\mathbf{E}^{(j)}(k)$ for user $j$ in Fig. 2 then equals the hermitian form of $\mathbf{A}^{(j)}(k)$ and the input of the FEC decoder can be described by

$$
\begin{aligned}
\hat{\mathbf{b}}^{(j)}(k) & =\operatorname{Re}\left\{\mathbf{E}^{(j)}(k) \cdot \mathbf{r}(k)\right\} \\
& =\operatorname{Re}\left\{\left[\mathbf{A}^{(j)}(k)\right]^{H} \cdot \mathbf{r}(k)\right\}
\end{aligned}
$$

The superscript []$^{H}$ denotes the conjugate transpose. Vector $\hat{\mathbf{b}}^{(j)}(k)$ at the FEC decoder input of user $j$ can be devided into three parts

$$
\hat{\mathbf{b}}^{(j)}(k)=\alpha(k)+\beta(k)+\eta(k) .
$$

The first term

$$
\begin{aligned}
\alpha(k) & =\left[\mathbf{A}^{(j)}(k)\right]^{H} \mathbf{A}^{(j)}(k) \mathbf{b}^{(j)}(k) \\
& =\left(\begin{array}{c}
\sum_{\mu=0}^{N_{p}-1}\left|H_{\mu}^{(j)}(k)\right|^{2} \cdot b_{1}^{(j)}(k) \\
\vdots \\
\sum_{\mu=(n-1) N_{p}}^{N_{c}-1}\left|H_{\mu}^{(j)}(k)\right|^{2} \cdot b_{n}^{(j)}(k)
\end{array}\right)
\end{aligned}
$$

represents the desired coded information obtained by maximum ratio combining (MRC) $N_{p}$ chips. The terms $\beta(k)$ and $\eta(k)$ describe the multiple access interference and the contribution of the background noise, respectively.

\section{Multi-User Detection}

\section{A. Linear MUD techniques}

Multi-user detection (MUD) schemes can be mainly devided into two groups, linear and nonlinear techniques [6]. Linear MUD schemes compute the pseudo-inverse $\mathbf{A}^{\dagger}(k)$ of the system matrix $\mathbf{A}(k)$ in (2) and thus perform a kind of equalization. It is necessary to make some comments on the calculation of the pseudo-inverse $\mathbf{A}^{\dagger}(k)$.

The system matrix $\mathbf{A}(k)$ consists of $N_{c}$ rows and $n J$ columns. Therefore, it describes a system of $N_{c}=n N_{p}$ linear equations with $n J$ unknown variables. If the number of users $J$ is larger than the CDMA spreading factor $N_{p}$, e.g. $J>32$ for $N_{p}=32$ and $n=2$, there are more unknown variables than equations and the linear equation system can only 
be solved with additional conditions. However, the pseudoinverse always exists and tries to find an approximation of $\mathbf{A}^{\dagger}(k) \mathbf{A}(k)=\mathbf{I}$ leading to an estimate $\hat{\mathbf{b}}(k)$ with minimum energy. For the case $J<N_{p}$, the pseudo-inverse has the form

$$
\mathbf{A}^{\dagger}(k)=\left(\mathbf{A}^{H}(k) \mathbf{A}(k)+\gamma \mathbf{I}\right)^{-1} \mathbf{A}^{H}(k)
$$

where $\gamma=0$ indicates the ZF equalizer (decorrelator) and $\gamma=$ $\sigma_{N}^{2}$ the MMSE solution. The term $\sigma_{N}^{2}$ represents the noise power [9]. For $J>N_{p}$,

$$
\mathbf{A}^{\dagger}(k)=\mathbf{A}^{H}(k)\left(\mathbf{A}(k) \mathbf{A}^{H}(k)+\gamma \mathbf{I}\right)^{-1}
$$

holds. The MMSE approach realizes a compromise between sufficiently decorrelating the interfering signals and noise suppression. Generally, the linear MMSE equalizer provides a performance improvement even in the case of $J>N_{p}$. There also exist sub-optimal reduced-rank approximations requiring less computational effort [9] but they are not considered here. The linearly filtered signal can be expressed by

$$
\begin{aligned}
\hat{\mathbf{b}}(k) & =\operatorname{Re}\left\{\mathbf{A}^{\dagger}(k) \cdot \mathbf{r}(k)\right\} \\
& =\operatorname{Re}\left\{\mathbf{A}^{\dagger}(k) \mathbf{A}(k) \mathbf{b}(k)+\mathbf{A}^{\dagger}(k) \mathbf{n}(k)\right\} .
\end{aligned}
$$

Due to the fact that FEC decoding is carried out after linear filtering, it is necessary to supply channel state information (CSI) to the FEC decoder. As stated before, OFDM offers the advantage that every chip is only affected by flat fading. Analyzing (10) for $J=1$ shows that a coded bit at the input of the FEC decoder can be expressed by

$$
\hat{b}_{i}(k)=b_{i}(k)+\frac{\operatorname{Re}\left\{\sum_{\mu=(i-1) N_{p}}^{i N_{p}-1} n_{\mu} H_{\mu}(k)^{*}\right\}}{\sum_{\nu=(i-1) N_{p}}^{i N_{p}-1}\left|H_{\nu}(k)\right|^{2}+\gamma}
$$

with $1 \leq i \leq n$. Although (11) does not hold any longer for $J>1$, an intuitive choice for channel state information is

$$
C S I_{i}^{(j)}(k)=\frac{1}{N_{p}} \sum_{\mu=(i-1) N_{p}}^{i N_{p}-1}\left|H_{\mu}^{(j)}(k)\right|^{2}+\gamma,
$$

i.e. $\hat{b}_{i}^{(j)}(k)$ is weighted with the sum of squared magnitudes of those channel coefficients associated with it.

Fig. 3 shows the results for a convolutional code with $L_{c}=$ 7 and $R_{c}=1 / 2$ and $J=16$ active users. It can be seen that the MMSE equalizer outperforms the ZF approach by $1.6 \mathrm{~dB}$. Without CSI, the MMSE approach loses up to $0.5 \mathrm{~dB}$ whereas the loss amounts approximately $2 \mathrm{~dB}$ for the decorrelator.

In Fig. 4, the influence of the MMSE filter on the desired signal is analyzed. The performance degradation for $J=16$ and $J=32$ users in comparison with the single user case is obvious. However, the reason for this degradation is not only

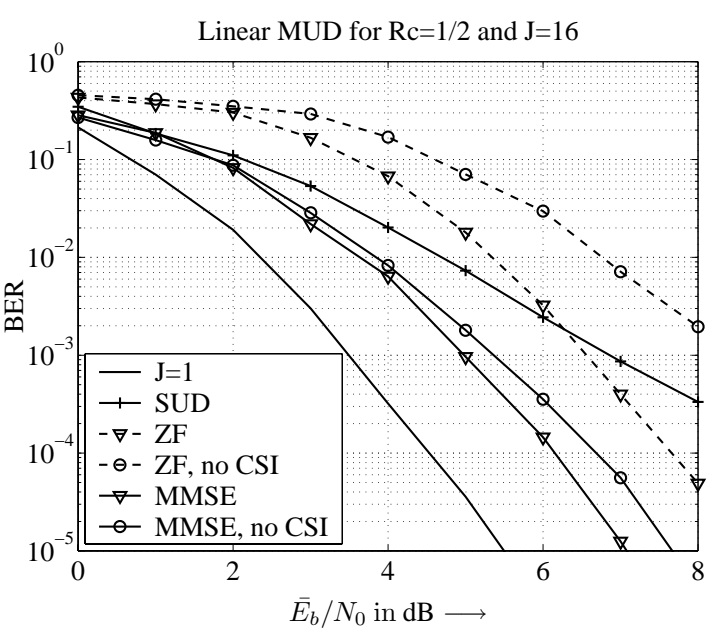

Fig. 3. BER for linear MUD for $J=16, G_{p}=64$

residual interference that has not been perfectly removed by the filter. In fact, a large portion of this impairment is caused by the insufficient equalization of the channel. In order to illuminate this effect, we carried out simulations where the interfering signals were ideally subtracted in front of the MMSE filter (dashed curves). Thus, the filter receives only the desired signal. From Fig. 4 we recognize that the performance loss for $J=16$ due to residual interference amounts only $0.5 \mathrm{~dB}$ whereas the loss compared to $J=1$ amounts $2 \mathrm{~dB}$. For $J=32$, the impairments due to residual interference is higher. However, its portion is still smaller than the degradation due to insufficient equalization. This observation has severe impact on the realization of the combined MMSE-PIC scheme described in III-C.

\section{B. Parallel Interference Cancellation}

Concerning nonlinear multi-user detection we regard the parallel interference cancellation (PIC) in this paper. Whereas successive interference cancellation is suitable for systems with large power variations of the received signals, PIC is predestinated for systems with strong power control. This ensures equal receive power of all users and all signals can be detected simultaneously.

The PIC procedure can be described in the following way. After individual SUD for each user, Soft-In/Soft-Out decoders deliver estimated information bits $\hat{d}^{(j)}(k)$ as well as loglikelihood ratios $L\left(\tilde{\mathbf{b}}^{(j)}(k)\right)$ of the coded bits [15], [16] Then, the expected values of $L\left(\tilde{\mathbf{b}}^{(j)}(k)\right)$ are calculated by the tanhfunction. Finally, the reconstructed signal $\hat{\mathbf{r}}^{(j)}(k)$ of user $j$ is obtained by $N_{p}$-fold repetition and scalar multiplication with the product $c_{\mu}^{(j)} H_{\mu}^{(j)}(k)$. The sum

$$
\tilde{\mathbf{r}}^{(j)}(k)=\sum_{i=1, i \neq j}^{J} \hat{\mathbf{r}}^{(i)}(k)
$$

over all interfering signals $\hat{\mathbf{r}}^{(i)}$ regarding user $j$ is now sub- 


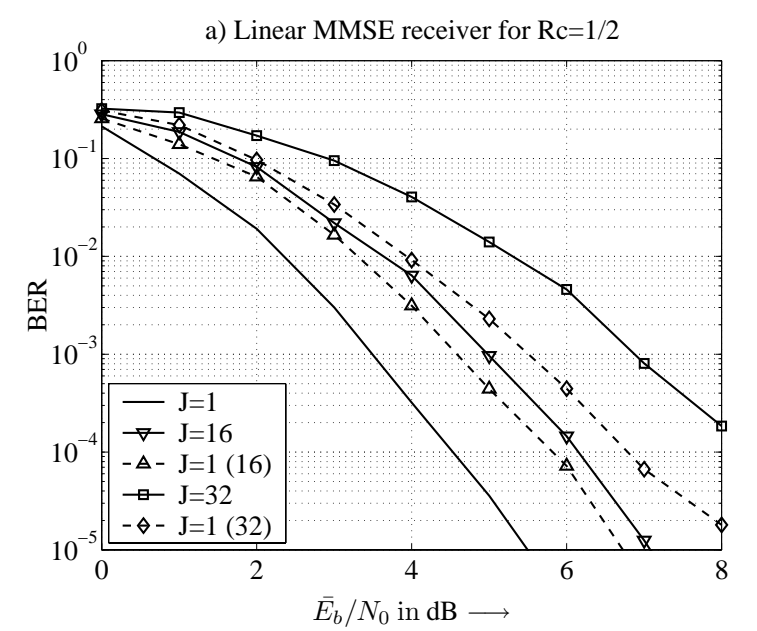

Fig. 4. BER for linear MMSE $(J=1(16)$ and $J=1(32)$ indicate the curves with perfect interference cancellation in front of the MMSE filter)

tracted from the received signal $\mathbf{r}$. In the absence of decoding errors, this difference is an estimate of the received signal of user $j$ without any multi-user interference. Therefore, passing this signal through the single-user detector and the channel decoder a second time should yield the performance of the single-user case. Due to decoding errors, the procedure described above has to be repeated several times.

The results for 3 PIC iterations are depicted in Fig. 5. It can be observed that, up to $J=32$, the single user performance (SUS) is reached. Note that $J=32$ active users lead to a system with the same spectral efficiency as half rate coded TDMA or FDMA systems. Increasing the number of users to $J=64$ leads to a tremendous loss because the signal-to-interference ratio equals $0 \mathrm{~dB}$ in this case. Regarding the performance of the considered convolutional code at $E_{b} / N_{0}=0 \mathrm{~dB}$ for $J=1$ indicates that the error rate at the decoder output is approximately 0.2. With this high error rate, the PIC scheme is not able to remove interference effectively and the iterative process does not converge.

\section{Combined MMSE and PIC}

From Fig. 5 it is obvious that solely parallel interference cancellation is not suited to remove MUI for high system loads, i.e. $J \geq G_{p}$. The initial performance of the error correcting coding scheme is not high enough to supply reliable estimates that can be used to re-construct the interfering signals accurately. Therefore, it might be advantageous to enhance the signal-to-interference ratio at the decoder input by replacing the single-user detectors by one MMSE multi-user detector.

Fig. 6 depicts one possible realization of the combined scheme. In a first stage, the MMSE multi-user detection is carried out increasing the SINR at the decoder inputs (switches in inner positions). Next, single-user FEC decoding is performed and the signals $\hat{\mathbf{r}}^{(j)}$ of each user are re-constructed and summed up according to (13). Due to the fact that the MMSE

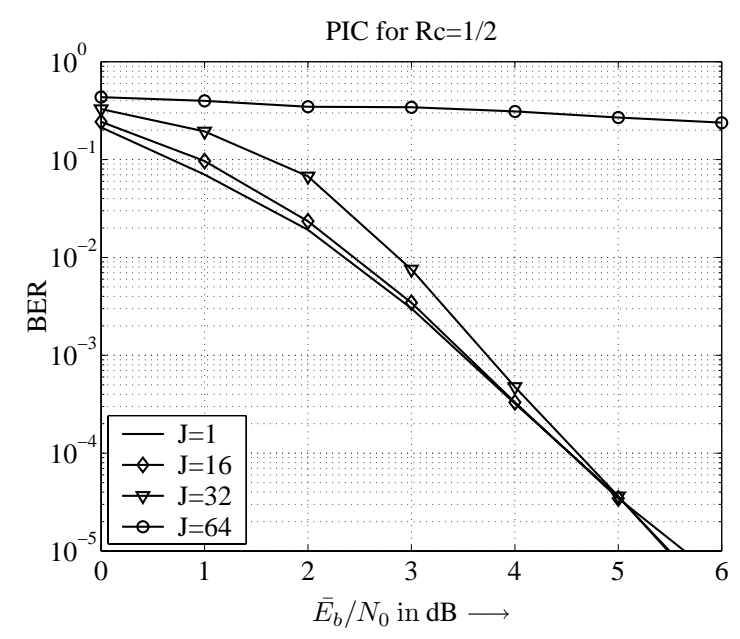

Fig. 5. BER for PIC with 3 iterations and different number of users

output suffers from an insufficient channel equalization (refer to section III-A) it is beneficial to use the MMSE filter only once for an improved starting point of the PIC loop. Therefore, the interference is subtracted directly from the received vector $\mathbf{r}$. In a second stage, the switches are turned to the outer positions and the interference reduced signals are single-user detected and decoded again several times according to section III-B. Thus, the MMSE filter is only working during an initial phase in order to improve the SINR at the decoder inputs.

In order to illuminate the importance of this arrangement, Fig. 7 shows the results for $J=32$ users. The curve labeled 'MMSE+PIC' represents a system where the MMSE filter is incorporated into the PIC loop and remains active during all iterations. As can be seen, the interference is totally removed by the PIC loop because the curve termed ' $J=1(32)$ ' is reached. However, there remains a gap of about $2,5 \mathrm{~dB}$ compared to the single user performance (see section III-A). If the PIC loop directly processes the received vector $\mathbf{r}$ as described in this section, we reach the single user curve (curve labeled 'MMSE-PIC').

A comparison of the different MUD schemes for $J=64$ active users is shown in Fig. 8. Obviously, the linear approach as well as the PIC scheme are not able to reduce MUI significantly. However, the combination of both asymptotically reaches the performance of a single-user system. Notice that the load of the system equals $J / G_{p}=1$ and is twice as high as the load of a conventional TDMA or FDMA system with half-rate FEC coding.

\section{CONCLUSION}

It has been shown that OFDM-CDMA offers great advantages for the application of multi-user detection in frequency selective environments. Due to flat fading on each subcarrier the computational costs for MUD are much lower than for comparable single-carrier systems. In coded systems, the use of channel state information improves the performance of $\mathrm{ZF}$ - 


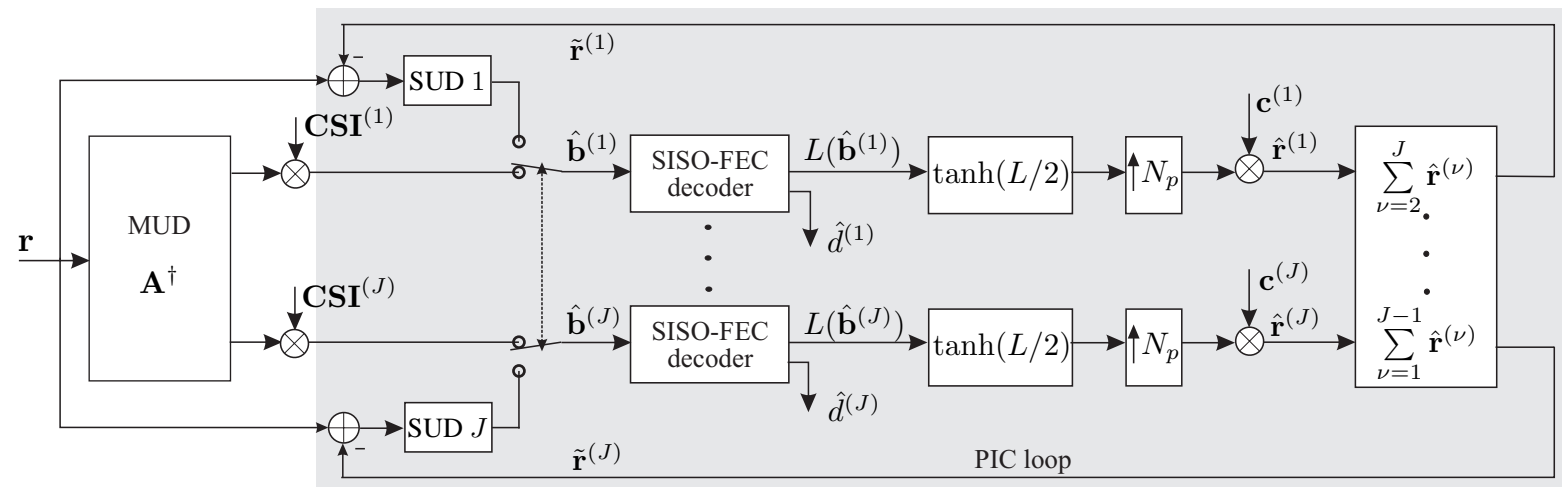

Fig. 6. Combination of linear MUD and parallel interference cancellation

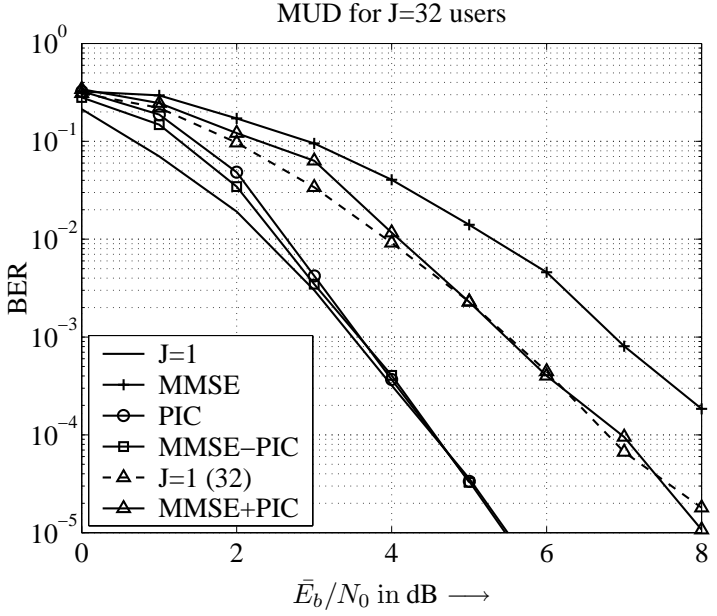

Fig. 7. Comparison of different MMSE-PIC combinations, 3 PIC iterations, $J=32$ active users

MUD by nearly $2 \mathrm{~dB}$ and by $0.5 \mathrm{~dB}$ for MMSE-MUD. Combining MMSE-MUD and PIC leads to a remarkable performance. If the MMSE filter is only used as a catalyst in an initial stage, the performance of a single-user system is reached for a bit error rate of $P_{b}=10^{-5}$ even for $J=G_{p}$.

\section{REFERENCES}

[1] E. Dahlman, B. Gudmundson, M. Nilsson, and J. Sköld. UMTS/IMT2000 Based on Wideband CDMA. IEEE Communications Magazine, pages 70-80, September 1998.

[2] T. Ojanperä and R. Prasad. An Overview of Air Interface Multiple Access for IMT-2000/UMTS. IEEE Communications Magazine, pages 8295, September 1998.

[3] A. Toskala, J. Castro, E. Dahlman, M. Latva-Aho, and T. Ojanperä. Frames FMA2 Wideband-CDMA for UMTS. European Transactions on Communications, 9(4):325-335, August 1998.

[4] Stefan Kaiser. Multi-Carrier CDMA Mobile Radio Systems - Analysis and Optimization of Detection, Decoding and Channel Estimation. $\mathrm{PhD}$ thesis, German Aerospace Center, VDI, January 1998.

[5] A. Dekorsy and K.D. Kammeyer. A new OFDM-CDMA Uplink Concept with M-ary Orthogonal Modulation. European Transactions on Telecommunications (ETT), 10(4):377-390, July/August 1999.

[6] S. Moshavi. Multi-User Detection for DS-CDMA Communications. IEEE Communications Magazine, pages 124-136, October 1996.

[7] S. Verdu. Multiuser Detection. Cambridge University Press, New York, 1998.

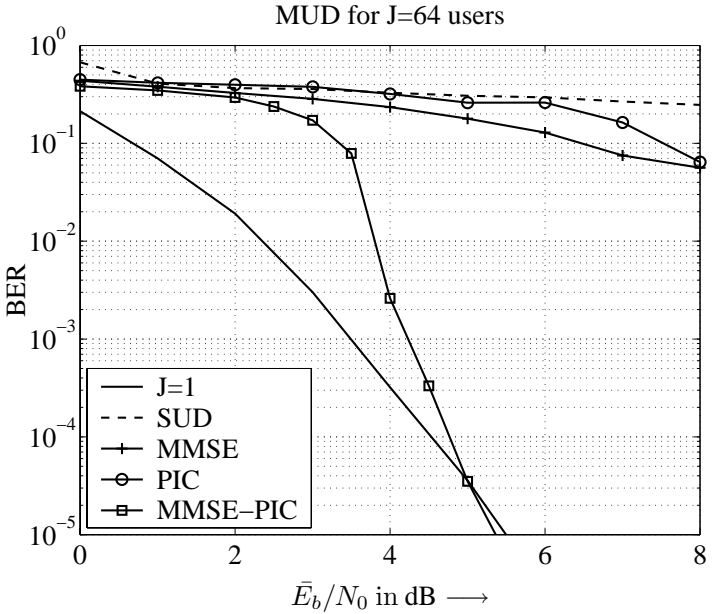

Fig. 8. Comparison of different MUD techniques for $J=64$ active users

[8] S. Verdu and S. Shamai. Spectral Ef£ciency of CDMA with Random Spreading. IEEE Transactions on Information Theory, 45(2):622-640, March 1999.

[9] M. Honig and M.K. Tsatsanis. Multiuser CDMA Receivers. IEEE Signal Processing Magazine, pages 49-61, May 2000.

[10] M.C. Reed, C.B. Schlegel, P.D. Alexander, and J.A. Asenstorfer. Iterative Multiuser Detection for CDMA with FEC: Near-Single-User Performance. IEEE Transactions on Communications, 46(12):1693-1699, December 1998.

[11] P.D. Alexander, M.C. Reed, J.A. Asenstorfer, and C.B. Schlegel. Iterative Multiuser Interference Reduction: Turbo CDMA. IEEE Transactions on Communications, 47(7):1008-1014, July 1999.

[12] P. Schramm and R.R. Müller. Spectral Ef£ciency of CDMA Systems with Linear MMSE Interference Suppression. IEEE Transactions on Communications, 47(5):722-731, May 1999.

[13] D. Tse and S.V. Hanly. Linear Multiuser Receivers: Effective Interference, Effective Bandwidth and User Capacity. IEEE Transactions on Information Theory, 45(2):641-657, March 1999.

[14] A. Klein. Multi-user detection of CDMA signals algorithms and their application to cellular mobile radio. $\mathrm{PhD}$ thesis, Düsseldorf: VDIVerlag, Fortschrittberichte VDI, Reihe 10, No. 423, 1996.

[15] V. Kuehn. Parallel Interference Cancellation in Coded DS-CDMA Systems. In COST 262 Workshop on Multiuser Detection in Spread Spectrum Communications, pages 31-38, Schloss Reisensburg, Germany, January 2001.

[16] V. Kuehn. Linear and Nonlinear Multi-User Detection in Coded OFDMCDMA Systems. In International Conference on Telecommications, volume 3, pages 239-244, Bucharest, June 2001. 